\title{
Iterative reconstruction techniques for X-ray microscopy in Geosciences
}

\author{
Matthew Andrew ${ }^{1}$, Shaun Graham ${ }^{2}$ and William Thompson ${ }^{1}$
}

1. Carl Zeiss X-ray Microscopy, 4385 Hopyard Rd, Pleasanton, CA

2. Carl Zeiss Microscopy, 509 Colhams Lane, Cambridge, UK

Non-invasive high resolution X-ray microscopy has found industrial application in a wide range of extractive industries. The Oil and Gas industry has seen a transformation of the application of pore scale imaging and modelling from a primarily research focussed pursuit focussed on the investigation of fundamental flow and transport properties in porous media to an increasingly crucial industrial tool for the characterization of a range of geological and petrophysical properties, including porosity \& pore connectivity, permeability, mineralogy, geomechanical response, geological facies type and diagenetic history [1]. In the mining industry, X-ray microscopy has the potential of transforming both laboratory and minesite analyses for determining properties such as modal mineralogy, assay, mineral association and liberation and particle and grain size distributions by extending these analyses (traditionally limited to 2 dimensional analytical techniques) into 3D (e.g. [2]).

One of the principal challenges when applying X-ray microscopy to solve industrial problems is that of throughput. High resolution X-ray microtomography acquisition times can be on the order of several hours, which can lead to challenging ROI calculations when weighing the relative advantage of high accuracy 3D analysis with cheaper, less capable analytical techniques. To tackle this issue optimization of each step in the production of the actionable information required by these industrial users is required. For 3D computed X-ray microtomography, these steps typically consist of sample mounting, scan setup, image acquisition, 3D computational reconstruction, image processing and segmentation and final analysis. In repetitive workflows (where many similar samples are run sequentially and image processing and analysis workflows are well understood), the slowest step is image acquisition.

Iterative reconstruction has the potential of greatly increasing reconstructed image quality for rapid acquisitions at low projection numbers or low exposure times, however several challenges must be overcome before it can be reliably used for industrial workflows. First of these is that it is vastly more computationally demanding than traditional analytical reconstruction techniques (i.e. FDK Filtered Back Projection), meaning that, while the acquisition stage of the workflow may be sped up dramatically, this comes at the cost of greatly increasing reconstruction times. Secondly iterative reconstruction requires the optimization of several tuneable parameters for effective reconstruction, which can be challenging for medium or low skill industrial operators.

In this paper we will showcase recent advances in the application of iterative reconstruction techniques, applied on the Versa 520 X-ray microscope (Carl Zeiss X-ray Microscopy). To solve the challenge of the computational cost of iterative reconstruction we implemented both algorithmic and computational optimizations. We use a statistical iterative algorithm including ordered subsets (OS) and Nesterov's momentum technique [3]. The combination of quadratic convergence rate provided by the momentum term, and additional linear convergence rate increase provided by OS reduce the number of iterations required for acceptable convergence from many hundreds to approximately 10. On the computational side, we use highly parallelized matched forward and back projection code implemented on nVidia GPUs using CUDA. 
We will then compare this implementation of iterative reconstruction to traditional analytical reconstruction for a homogenous reservoir sandstone (Oil and Gas) and a mine tailing sample. A comparison will be made both of quantitative metrics of image quality (signal to noise ratio and edge sharpness) and analyses of actionable information. The reservoir sandstone images were then processed and segmented for permeability simulation, and the mine tailing images were processed to give grade vs. recovery and liberation vs. recovery information. Both application show that, for a defined processing and segmentation workflow, equivalent results can be obtained using as few as much one quarter the projections (and so one quarter the scan time) commonly used in such scans.

[1] MJ Blunt et al. Advances in Water Resources 51 (2013), p. 197-216

[2] Ghorbani et al. Minerals Engineering 24 (2011), p. 1249-1257

[3] D Kim, S Ramani and JA Fessler. IEEE Transactions on Medical Imaging 34:1 (2015), p. 167-178
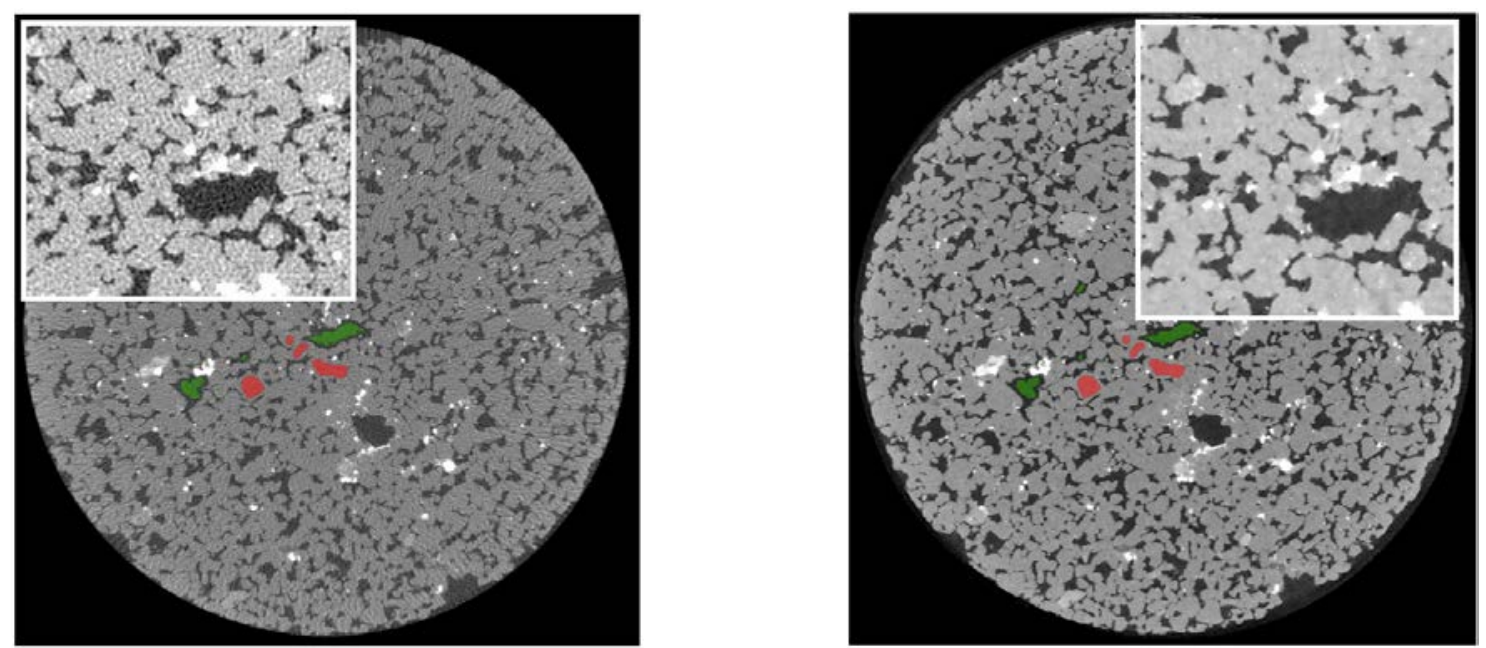

Figure 1. Comparison results of analytical (filtered back projection) reconstruction (left) and iterative reconstruction (right) for a sandstone sample reconstructed from a dataset of only 400 projections.
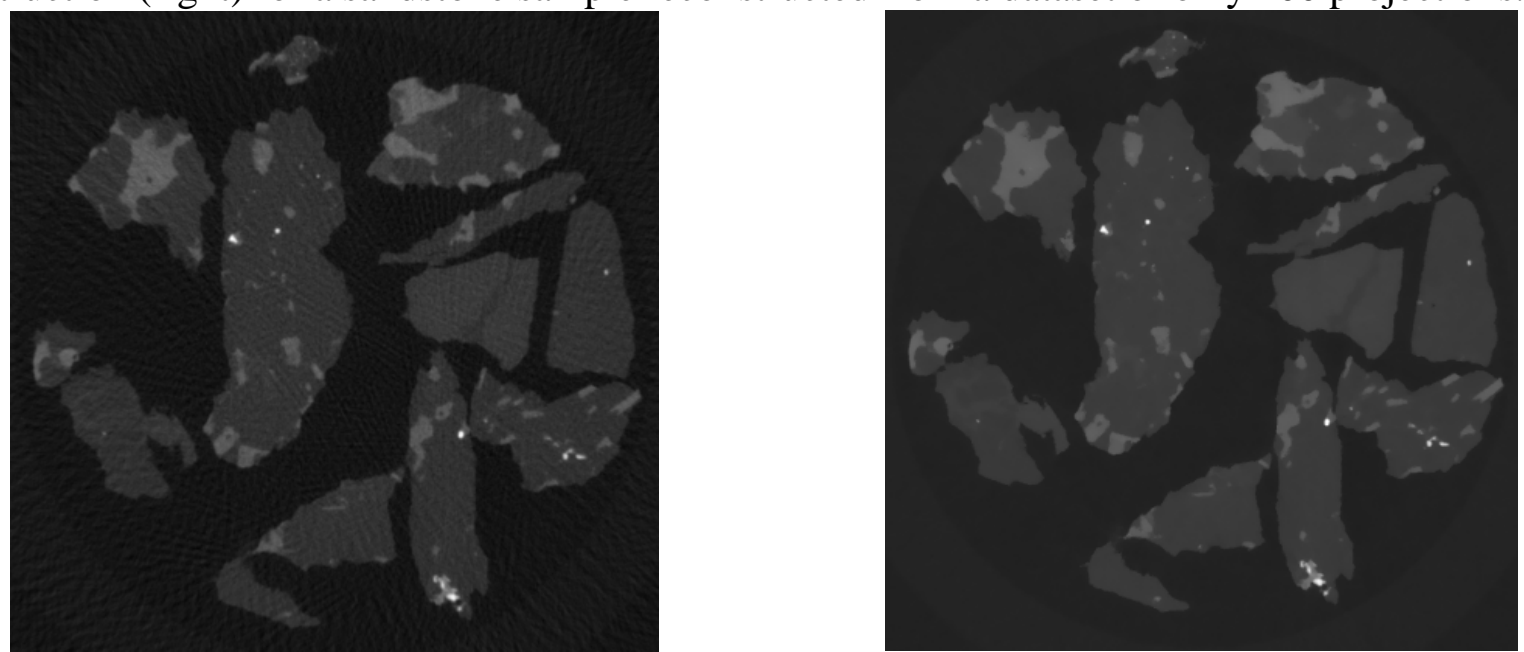

Figure 2. Comparison results of analytical (filtered back projection) reconstruction (left) and iterative reconstruction (right) for a sample of mine tailing reconstructed from a dataset of only 200 projections. 